\title{
DEL TEXTO A LA CULTURA. APUNTES SOBRE EL PENSAMIENTO SISTÉMICO APLICADO A LOS ESTUDIOS CULTURALES
}

\author{
FROM TEXT TO CULTURE. NOTES ON SYSTEMIC THINKING \\ APPLIED TO CULTURAL STUDIES
}

\author{
Mirko LAMPIS \\ Universidad Constantino el Filósofo de Nitra (Eslovaquia) \\ mlampis@ukf.sk
}

Resumen: En este artículo se propone un análisis sistémico de las nociones de «texto»y «cultura». Más concretamente, gracias a teorías como la Semiótica de la Cultura, la Teoría de los Polisistemas y la Sociocrítica, se pone de relieve la dimensión sistémica que caracteriza a los fenómenos y procesos culturales.

Abstract: This paper proposes a systemic analysis of notions of «text» and «culture». More specifically, through theories such as Semiotics of Culture, Polysystem Theory and Sociocriticism, it highlights the systemic dimension that characterizes the cultural phenomena and processes. 
Palabras clave: Texto. Cultura. Semiosis. Pensamiento sistémico.

Key Words: Text. Culture. Semiosis. Systemic thinking.

1. Hablando de textos y de análisis textual, a pesar de la enorme variedad de posturas críticas y matices teóricos existentes, contamos con al menos dos afirmaciones acerca de las cuales el acuerdo de los especialistas parece ser prácticamente unánime. Con respecto a un texto dado ${ }^{1}$, se suele admitir: 1) que no existe una única interpretación, una única lectura universalmente válida (una Verdad totalmente inmanente al texto) y 2) que no todas las lecturas posibles son igual de legítimas, es decir, que a partir de la estructura material, la función y la historia del texto existen interpretaciones viables (o correctas) y existen errores y excesos interpretativos, cuando no verdaderos abusos textuales ${ }^{2}$. Naturalmente, estas dos afirmaciones presuponen e implican otra aún más fundamental: algo es un texto si y sólo si alguien así lo interpreta a partir de otros modelos textuales ya aprendidos.

2. La relación entre texto y cultura no es unidireccional, en el sentido de que reitera e invierte constantemente el proceso de definición: por un lado, el texto es el producto de una actividad cultural, el resultado de un proceso creativo que se inscribe en un dominio de relaciones culturales (desde este punto de vista, la cultura define lo que es o no es un texto); por otro, el texto, en tanto que producto y expresión activa en la red cultural, perpetúa y modifica los equilibrios internos de esta red, convirtiéndose, por ende, en un agente modelizante, reconstructor y ordenador de determinados sistemas de relaciones culturales (en este sentido, el texto define lo que es y cómo es la cultura $^{3}$ ). Si la cultura, en otros términos, funciona como un sistema de producción, circulación y memoria textual (Lotman, 1996), sus intérpretes (ac-

\footnotetext{
${ }^{1}$ Podemos definir el texto, en términos generales, como un conjunto integrado de expresiones al que se asigna o en el que se reconoce una determinada organización significante. Por conjunto integrado, entenderemos un conjunto cuyos elementos interactúan de forma recursiva, de modo que existen o se conocen sólo en virtud de su mutuo interactuar; por expresión, un evento (o cambio) pertinente y significante en el flujo cognoscitivo; por organización, el conjunto de aquellas relaciones internas sin las que el sistema no existiría o no se reconocería como tal.

${ }^{2}$ Como también ha señalado Umberto Eco, hasta un desconstruccionista radical que defienda la absoluta arbitrariedad o coyunturalidad de toda interpretación pretende luego que lo que él afirma y sostiene se interprete de una manera determinada, y no de cualquier modo.

${ }^{3}$ Este hecho resulta particularmente evidente en el caso de culturas alejadas en el espacio y en el tiempo, culturas que a veces conocemos sólo a través de unos pocos fragmentos textuales. En cualquier caso, toda cultura establece un modelo de sí misma al seleccionar y fijar un canon regular y estable ("impermeable") de textos de referencia y de interpretaciones correctas o incluso un único Texto depositario de sus principios y hábitos fundamentales.
} 
tores, lectores y traductores) sólo pueden conocerla y participar en ella a través de sus textos. La cultura se nos presenta como un sistema textual circuital, tautológico, autorreferente.

3 . Es la actividad y el mutuo interactuar de los intérpretes lo que determina el funcionamiento de los textos, sus concretas modalidades de activación y actualización; asimismo, es la interacción con y mediante los textos lo que regula y guía el aprendizaje de los intérpretes y, por tanto, la formación de sus hábitos significantes y comunicativos ${ }^{4}$. Textos e intérpretes, en suma, se codefinen, coparticipan y derivan en un único dominio de interacciones e historias culturales, y es únicamente en este dominio donde pueden surgir, perdurar e innovarse determinadas tradiciones e intenciones textuales.

Así pues, si podemos acercarnos a la intentio operis (como diría Eco), si podemos reconocerla, si podemos reconstruirla y comunicarla, si podemos colaborar con el texto, es sólo porque partimos de la estructura material (o expresiva) del texto en relación con su contexto y su historia, es decir, el dominio operacional que define y suporta dicha estructura y nuestra relación con ella. Un dominio operacional, no lo olvidemos, que está integrado por numerosos agentes (individuales, colectivos, institucionales) que se mueven, operan y derivan en diferentes planos o redes relacionales (diacrónicas, sociales y pragmáticas), incluyendo también todos aquellos ámbitos (científicos o no) en los que el texto se vuelve objeto de una cura o atención descriptiva especial finalizada a la fijación canónica e institucional de su significado.

Si la antigua imagen de una divinidad ahora expuesta en un museo, según señalaba Gadamer (1983: 9), al lado de una fascinación estética que antes, en su templo, no ejercía todavía, nos recuerda el mundo de religiosidad del que procede, es porque aquel mundo es todavía parte de nuestro mundo. Porque, diremos, disponemos de interpretantes (y de textos) que aún nos permiten pensar en, e intentar reconstruir, el sistema de creencias que motivó al autor y a los antiguos receptores de la imagen o, en otras palabras, porque en nuestro dominio cultural, junto a la imagen, han persistido aquellas relaciones significantes (aquellos hábitos interpretativos y aquel interés) que sustentan una lectura de tipo religioso.

4. Este enfoque de análisis que intenta esclarecer las relaciones que se dan entre el texto y su dominio de existencia (sincrónico y diacrónico) se

${ }^{4}$ Como a menudo ha señalado Lotman, si, por un lado, un texto es siempre el producto de una actividad lingüística (un texto en alguna lengua), por otro, el texto precede al lenguaje, y lo genera (el lenguaje sólo nace y se define a partir de la interacción textual). 
puede definir, de manera muy general y a falta de denominaciones más precisas, con una fórmula que ya se está empleando en otros muchos ámbitos científicos: «enfoque sistémico», o «relacional», o «complejo».

Se trata, sustancialmente, de volver a colocar el texto (y su significado) en la trama de relaciones culturales e históricas en que éste participa y cobra relevancia; de escarbar en las relaciones no sólo internas al texto, sino también en aquellas relaciones intertextuales que lo identifican y sostienen; de individuar, en cada caso concreto, el papel del texto en su contexto y del contexto en el texto; de hacer hincapié en el hecho de que toda lectura (canónica, viable o revolucionaria) se produce en, y es solidaria con, un momento y unas circunstancias históricas determinadas.

5. Hallamos, en efecto, elementos, reflexiones y aspiraciones de tipo sistémico en casi todas las teorías literaturológicas y culturológicas contemporáneas, incluso en las teorías más analíticas, con aspiraciones más claramente reduccionistas, como pueden ser las de Hjelmslev o de Greimas. El caso de Hjelmslev resulta particularmente interesante. El poderoso y detallado aparato analítico que el lingüista danés intentó desarrollar encuentra su necesario complemento en la noción de catálisis: no una partición del objeto examinado en constituyentes más simples, sino la recomposición del objeto con otros objetos en una clase analítica más amplia y articulada; la entera serie analítica, precisa Hjelmslev, puede ser así concebida como una catálisis que individúa y conecta una sintagmática concreta (una estructura) a una paradigmática (un sistema de significado) (Hjelmslev, 2009).

Existen, además, y quizás sea éste el dato más importante, teorías que acogen y reflejan las propias especificaciones fundamentales del pensamiento sistémico ${ }^{5}$. Podemos citar la Teoría de los Polisistemas del profesor Itamar

5 Tales especificaciones se pueden resumir de la siguiente manera: 1) el pensamiento sistémico se ocupa de totalidades, redes integradas cuyos componentes generan, a través de sus interacciones recursivas, un comportamiento de alto nivel que modifica y condiciona el operar y la deriva de cada componente; 2) los objetos de que se ocupa la investigación son conjuntos integrados de relaciones cuyas fronteras varían (cuya identificación varía) según las relaciones consideradas; estos objetos no sólo son, sino que también participan en redes de relaciones, y su valor para la investigación estriba, precisamente, en las relaciones en que participan; 3) las relaciones de las que se ocupa el pensamiento sistémico son relaciones dinámicas, relaciones que cambian en el tiempo y según las condiciones de contorno; son, en otros términos, procesos históricos; 4) el investigador-descriptor no es nunca un elemento externo a la red que describe; su actividad es parte integrante del sistema y contribuye por tanto a delimitar, ratificar o rectificar las relaciones que lo identifican y definen; 5) el investigador-descriptor no puede conocer ninguna realidad objetiva e independiente de lo que él es y hace: todo conocimiento depende de la legalidad estructural y operacional del sujeto cognoscente, legalidad determinada por la deriva orgánica, relacional y social del propio sujeto. 
Even-Zohar, de la Universidad de Tel Aviv, acaso la teoría que mejor y más claramente explicita sus principios sistémicos. La Sociocrítica del profesor Edmond Cros, de la Universidad «Paul Valéry» de Montpellier, un intento de dirigir el análisis estructural hacia la individuación de aquellas huellas textuales que remiten a las diferentes formaciones ideológico-discursivas que constituyen el sustrato histórico y colectivo del texto. Y la Semiótica de la Cultura de Iuri M. Lotman, una poderosa reflexión sobre los procesos de interdependencia, la heterogeneidad semiótica y los fenómenos creativos e imprevisibles que caracterizan al dominio textual de la cultura (Lampis, 2011).

6. Las fronteras de un texto no son un espacio perfectamente delimitado. Es decir, la identificación de un texto, en pos o en contra de cierta tradición, responde siempre a una determinada (e históricamente contingente) estrategia cognoscitiva. La estructura material del texto, su forma y sustancia expresiva, está sometida, por decirlo de alguna manera, al «arbitrio» de las comunidades autoorganizadas de intérpretes (actores, lectores y traductores). También cuando en tales comunidades existe y se impone el escrúpulo filológico y crítico de respetar (y recuperar, si hace falta) una determinada forma canónica o una tradición hermenéutica pretérita.

Si la Divina Comedia, La Rendición de Breda o Los sietes samuráis dicen lo que dicen y enseñan lo que enseñan, es porque aprendimos y perpetuamos el hábito de considerarlos como entidades estables que conservan ciertas propiedades salientes aun cuando se reproducen, se citan, se resumen, se fragmentan en partes, se relacionan con otros textos y participan en todo tipo de historias y de vidas. Pero piénsese en una divina comedia oral, transmitida de boca en boca y con licencia para elegir, combinar y modificar según el gusto de cada narrador, o en una rendición de Breda entregada a un colectivo de niños de primaria armados de pinceles, o en un juego de rol donde cada samurái es siempre una persona distinta que puede modificar el carácter y las acciones de su personaje. Cambiaría ciertamente, en estas condiciones, la estabilidad y unidad «filológica» de los textos y, sin embargo, seguiríamos hablando de textos organizados de cierta forma, de juegos que admiten sólo determinadas reglas de conducta.

7. Todo proceso de autoorganización conlleva ligaduras y constricciones operacionales, de modo que el «arbitrio» de una comunidad de intérpretes nunca es tal en términos absolutos. Quien sostiene que la Divina Comedia narra la historia del viaje ultraterreno de dos amantes, Dante y Virgilio, tiene todo el derecho de creerlo así, si así lo quiere, pero está solo. Porque la Divina Comedia no es sólo el producto de un determinado proceso creativo y de 
un incalculable número de operaciones interpretativas, sino también una estructura estable, canonizada, un texto que desempeña una función activa en la propia red de relaciones (y autoorganizaciones) culturales. Cada cual puede jugar con el texto, según sus competencias y gustos, pero a la hora de conversar acerca de ese mismo texto, a la hora de definir un dominio viable de comunicación, priman determinadas interpretaciones socialmente pertinentes, resultado de un proceso histórico de sedimentación y de criba de significados.

Si algún conocido me dice que mañana tendremos lluvia, gracias a mis conocimientos de la lengua, de la metereología y del estado actual del cielo podré declararme o no de acuerdo con él, o zanjar la cuestión con un clásico «¿cree usted? Pues no habrá que olvidar el paraguas». Pero resultaría cuando menos insólito que le contestara a tan inocente afirmación con enfado y hasta con insultos. A no ser que mi universo de significado fuera total o parcialmente desligado del dominio de interacciones sociales que me rodea (un fenómeno que se suele indicar con expresiones como «enajenación mental», «trastorno cognitivo»o «esquizofrenia»).

8. Naturalmente, el texto «pienso que mañana lloverá» es de una linealidad tal y tiene una génesis tan trivial que (normalmente) no presupone ninguna dificultad y por lo tanto ningún esfuerzo interpretativo; en cambio, obras como la Divina Comedia, el Ulises o el Mahabarata, por complejidad, génesis e historia, suelen implicar la activación de múltiples e intricados trayectos de sentido. Sin embargo, a pesar de esta diferencia fundamental, en ninguno de los dos casos se puede pasar por alto el peso de la tradición, de los hábitos de «lectura» adquiridos.

Considérense las tres siguientes afirmaciones: a) en este local hay mucho ruido; b) las ondas sonoras se desplazan con una velocidad que depende de las características del medio físico de propagación; c) qué ruido tan triste el que hacen dos almas cuando se aman. Al leerlas, es casi inmediata su identificación y diferenciación mutua: simple constatación la primera, explicación científica la segunda y... ¿la tercera? Es bien posible que esta última afirmación despierte cierta perplejidad; o puede que se califique en seguida como expresión literaria; e incluso que se reconozca como el primer verso de unos de los poemas que componen Los placeres prohibidos (1931), de Luis Cernuda ${ }^{6}$.

${ }^{6}$ Pero no es un verso de Cernuda, ya que el poema de este autor empieza, en realidad, así: "Qué ruido tan triste el que hacen dos cuerpos cuando se aman". ¿Ha pasado desapercibida la sustitución de "cuerpos" por "almas"? ¿Ha molestado? ¿Y si dijera que tengo en mi poder una versión autógrafa del autor, fechada a 22 de octubre de 1948, en la que aparece el término "almas" en lugar de "cuerpos”? ¿Cambiaría algo? ¿Y es correcto, al fin y al cabo, o al menos incuestionable sostener que el poema es del autor que lo crea? 
Diremos que el hecho de interpretar algo como un texto o parte de un texto y de catalogarlo en (relacionarlo con) un registro, estilo o dominio expresivo concreto depende, en último término, de nuestra competencia cultural, de lo que hemos aprendido, de la capacidad adquirida para reconocer y producir determinadas expresiones en determinados contextos. Todos sabemos, por ejemplo, que para interpretar un texto escrito en una lengua dada es imprescindible saber leer en esa lengua. Pero saber leer puede no ser suficiente si esta actividad no se fomenta y afina gracias a determinados hábitos de lectura. No se le obliga a un niño de nueve años a leer Guerra y paz, y un lector acostumbrado sólo a los últimos best-sellers de moda con toda probabilidad encontrará acongojante (en el mejor de los casos) la lectura de una novela de Joyce o de Faulkner.

Dar sentido a un texto significa conectarlo a una memoria textual determinada. Más concretamente, a una memoria donde las expresiones y procesos que integran el texto no caigan como semillas en el desierto.

9. La memoria a la que el texto se conecta no se limita a la memoria biológica del intérprete (sus recuerdos, sus conocimientos), sino que se extiende hasta abarcar todas las prácticas y hábitos significantes que integran el dominio social en que el intérprete participa y deriva. Una memoria textual es siempre una memoria compartida.

Ya lo escribió con bastante claridad Gadamer al investigar la noción heideggeriana de círculo hermenéutico: el comprender (comprender algo como un texto y comprender el significado de un texto) ha de entenderse, más que como una actividad individual del sujeto, como una participación activa en un proceso de transmisión histórica (en una tradición de sentido); entender el significado de las partes y relaciones que integran un texto implica necesariamente una labor que se desarrolla a partir de ciertas expectativas de sentido, las cuales se derivan de un contexto precedente; así pues, la interpretación busca un acuerdo entre las partes, los particulares del texto, y el todo al que estos particulares se relacionan: si dicho acuerdo falla, la interpretación no funciona y ha de ser abandonada (Gadamer, 1983: 340-341). Y también Umberto Eco: cualquier intento de aislar y definir una forma significante necesariamente comporta el reconocimiento previo de su pertinencia con respecto a una hipótesis global que se adelanta a (y justifica) la propia práctica descriptiva: no puede haber análisis de rasgos significantes que ya no implique una interpretación, un relleno de sentido (Eco, 1979: 10). Un relleno de sentido, no está de sobra repetirlo, que se deriva de las especificaciones y expectativas de unos hábitos interpretativos aprendidos en un contexto cultural determinado. 
Muy acertada, por lo tanto, nos resulta una de las conclusiones del ya citado Gadamer: comprender un texto no significa ni ajustar lo que sabemos a lo que el texto dice ni ajustar lo que el texto dice a lo que sabemos; es, más bien, un diálogo que busca un encuentro, un camino cognoscitivo viable, el deslumbramiento de lo que texto e intérprete tienen en común en tanto que participantes en una misma historia cultural.

10. El hecho de considerar la cultura como un sistema (o repertorio) textual autoorganizado y autorreferente no significa pensarla y describirla como un bloque homogéneo cuyas fronteras constituyen auténticas superficies de ruptura, barreras que separan netamente un dentro de un fuera. La cultura es, más bien, un dominio integrado de interacciones comunicativas y significantes, un dominio que, en palabras de Cros (2009: 162), no existe sino en la medida en que se diferencia de otros dominios y cuyos límites son acotados por un sistema de índices de diferenciación.

Naturalmente, sostener que el establecimiento y la defensa de unas fronteras culturales concretas dependen de unos criterios de diferenciación (lingüísticos, políticos, tecnológicos, geográficos, temporales, etc.) equivale a decir que la extensión en el espacio y en el tiempo de cualquier dominio cultural varía según los procesos integrativos considerados por quienes establecen la distinción. Las fronteras entre culturas diferentes y entre cultura y espacio extra-cultural no serían nunca, por consiguiente, líneas perfectamente delimitadas e infranqueables, sino umbrales de intensidad y recursividad interaccional.

Así pues, dado un dominio cultural determinado, es legítimo defender tanto su fragmentación interna como su participación en un dominio cultural más extenso. Por un lado, toda cultura es heterogénea, atravesada ella misma por fronteras y umbrales, por diferentes flujos de organización semiósica (por ello, los sujetos culturales pueden participar y realizarse en diferentes ámbitos de significado, simultánea y sucesivamente). Por otro lado, las diversas culturas se relacionan e interdefinen, hasta llegar a la cultura como fenómeno global, como semiosfera, según la conocida fórmula lotmaniana: el espacio-tiempo de todos los procesos semiósicos, el dominio cognoscitivo y la historia cognoscitiva en que se mueven, operan e interactúan todos los seres humanos y todos sus textos. Un dominio y una historia que ya han alcanzado una dimensión planetaria.

11. La heterogeneidad o, si se prefiere, la fragmentariedad de todo dominio cultural de ninguna manera constituye un defecto o una disfunción que limita o invalida el operar de la cultura. Vale, más bien, el contrario (otra lección que debemos a Iuri Lotman): sin heterogeneidad, la cultura no existiría. 
Los textos semióticamente complejos y los dominios semiósicos complejos son necesariamente heterogéneos. Algo que ya expresó con mucho acierto Miguel de Cervantes en el prólogo de la segunda parte de su Quijote. Entre los muchos comentarios que Cervantes dedica al anónimo autor del «Segundo Don Quijote», hallamos el siguiente: «Pero, en efecto, le agradezco a este señor autor el decir que mis novelas son más satíricas que ejemplares, pero que son buenas; y no lo pudieran ser si no tuvieran de todo». Pues bien, «tener de todo», es decir, en términos lotmanianos, la coexistencia (más o menos conflictiva) de lenguajes diferentes, constituye una condición imprescindible para que un sistema semiótico pueda producir sentido.

Las dinámicas que se dan entre lo conocido y lo desconocido, entre lo que el intérprete sabe y lo que descubre, entre lo que reconoce por hábito y lo que tiene que integrar mediante alguna hipótesis (que queda a la espera de ulteriores confirmaciones... o de una rectificación), las dinámicas, en suma, mediante las cuales se modifica el dominio cognoscitivo del intérprete y el dominio de pertinencia del texto, dependen, en última instancia, de los procesos significantes que se activan y derivan a partir de la propia interacción textual. Así pues, la presencia simultánea de diferentes modalidades de interpretación, de diferentes prácticas textuales, de diferentes hábitos significantes, su presencia tanto en el repertorio cultural dado como en la memoria del intérprete y en la propia estructura del texto, garantiza que las labores de interpretación, transcodificación y traducción generen soluciones de sentido imprevisibles y, por ende, que los procesos del conocimiento no se estanquen en una rutina, un método o un procedimiento constante y universalmente válido.

12. Las diferencias, discrepancias e incomprensiones que puede generar la heterogeneidad (o semiodiversidad) cultural resultan productivas si y sólo persiste una voluntad de comprensión (comprensión, no acuerdo) y por lo tanto cierta disponibilidad al diálogo y a la conversación. Una disponibilidad que, en sus diferentes formas y gradaciones, caracteriza a la existencia cultural en cuanto tal.

Desligado y alejado de un mundo lineal de significado como consecuencia de la propia emergencia de la semiosis, al ser humano en su existencia individual y colectiva no le quedan más opciones que la de proseguir en una búsqueda constante (e imperfecta) de sentido. Y es importante entender que todas las soluciones cognoscitivas a las que conduce esta búsqueda, aun en los casos extremos del nihilismo (nada tiene sentido) y del fa- 
natismo (ésta es la Verdad, todo lo demás es error, herejía, pecado), pueden subsistir y derivar como soluciones viables sólo si resultan congruentes con el contexto cultural, es decir, sólo si participan en algún proceso dialógico y conversacional que pueda legitimarlas.

13. Humberto Maturana (2009) sostiene que lo que motiva y distingue a la práctica científica es, primariamente, una emoción o pasión ${ }^{7}$ específica que él define como pasión por explicar. Podemos añadir que esta pasión o emoción de fondo se define a partir de la curiosidad, o actitud exploratoria, que caracteriza a todos los mamíferos y que, en el linaje de los homínidos, llega a nuevas e imprevisibles modalidades operacionales con la explosión de la semiosis. También podemos añadir que la pasión por explicar, por construir redes de significados, por conectar experiencias, emociones y flujos comunicativos (lo que el propio Maturana llama «conversar»), caracteriza no sólo a las prácticas científicas, sino a todos los procesos de la cultura. Y que, naturalmente, dada la dimensión social e histórica de tales procesos, resultaría incomprensible si la desvinculáramos de las condiciones que la sostienen en términos, sobre todo, de tradición y validación.

Simplificando, diremos que el sujeto cognoscente, al participar en una tradición, entra en contacto con unos textos que quiere explicar mientras aprende de los demás y conversa con los demás acerca de la legitimidad de las explicaciones que le permiten operar con esos mismos textos.

14. En el sistema de la cultura, cualquier variación (observada) en los procesos de producción y de interpretación textual resulta significativa y se convierte, como diría Bateson (2000), en información. Información acerca de las dinámicas del flujo comunicativo. Información sobre la estructura y el funcionamiento semiótico del texto. Sobre su génesis. Sobre su historia. Sobre el contexto de interpretación. Sobre los propios intérpretes.

El flujo informativo es, pues, multidimensional y continuo, y sus dinámicas son tales que sólo por comodidad explicativa, heurística o analítica se pueden aceptar distinciones ya clásicas y fuertemente institucionalizadas como la distinción entre sintaxis, semántica y pragmática o entre sincronía y diacronía. No hay que olvidar, sin embargo, más allá de toda conveniencia y convención, la profunda unidad que se da entre estructuras, correlaciones y prácticas significantes y entre invariancias y derivas textuales.

${ }^{7}$ Una emoción es una disposición a la acción que surge como consecuencia del propio operar (y de la propia deriva) del organismo. En este caso concreto, el término pasión se emplea como sinónimo, aun imperfecto, de emoción, e indica por tanto un sentir dirigido a la acción en un dominio operacional dado. 
Dijimos antes que toda creación y todo reconocimiento arrancan necesariamente de una tradición, de unos hábitos cognoscitivos, de una memoria. Pero dicha memoria no funciona como un archivo o repertorio del que se puedan sacar piezas y moldes prefabricados y preordenados para el uso. La memoria (la memoria textual de una sociedad y la memoria biológica de sus miembros) es un sistema dinámico en continua e imperfecta reconstrucción, en perpetua deriva. Por ello, fenómenos tan dispares como los recuerdos, las valoraciones de los hallazgos científicos, las discusiones acerca del canon o la interpretación de una obra de arte presentan un importante punto en común: no conluyen. La dimensión ilimitadamente recursiva de la semiosis, la heterogeneidad de los ámbitos de producción e interpretación textual y la variedad de los procesos de conversación y validación cultural hacen que tanto el texto como la memoria que lo activa cambien a lo largo de su interacción.

Así pues, tanto la creación de un texto, a partir de un intertexto dado (o universo ideológico-discursivo) como la interpretación de ese mismo texto a partir de otros intertextos (otros universos ideológico-discursivos con los que el texto puede entrar en contacto por tradición, transferencia, accidente) implican necesariamente la aplicación de operaciones aprendidas y también un juego de recombinaciones, hipótesis y apuestas más o menos atrevidas o innovativas ${ }^{8}$. Así, la práctica textual se resuelve en un complejo hábito cognoscitivo (semiósico) que lleva en sí las condiciones de su propia transformación. Así, el significado y el valor de un texto dado se desplazan (derivan) según el contexto en el que el texto participa a la vez que contribuye a construir ese mismo contexto ${ }^{9}$. Así, la cultura sienta las bases de lo que Lotman acertó a definir como una de sus funciones fundamentales (junto a la comunicación y a la memoria): la creación constante de nuevos textos (textos

\footnotetext{
${ }^{8}$ Naturalmente, el reconocimiento y la valoración de cualquier novedad sólo se pueden dar a partir de lo ya conocido.

9 Éste es un dato que cualquier estudio literario o cultural puede, directa o indirectamente, confirmar. Refiriéndome sólo a los estudios especializados que están a mi más inmediato alcance, puedo citar: las dinámicas culturales que se establecen entre el uso de determinadas prendas (o textos indumentarios), los vocablos que las designan, la identificación y valoración social que las acompaña y los juegos metonímicos y metafóricos que originan (Štrbáková, 2011); las constricciones que imponen y los cambios y actualizaciones que sufren los elementos narrativos que identifican al mito del bandolero en una producción teatral y un mercado fuertemente codificados como los de comienzos del siglo XX (Bojničanová, 2009); los efectos (a la vez de "mímesis" y de "extrañamiento") debidos a la incorporación de "lenguajes-otros", de lenguas (y variedades de lengua) que identifican al otro, en la estructura unitaria de una obra de ficción (Pappová, 2008); la narración como necesidad orgánica en la experiencia vital y cultural del escritor, experiencia que luego modifica al propio flujo narrativo (Kučerková, 2011).
} 
impredecibles). Función que en parte pueden controlar (y a menudo limitar) los sectores hegemónicos de la cultura, pero que es ineliminable del hecho cultural en cuanto tal.

15. La cultura nació, según Levi Montalcini (1999), cuando los miembros de las tribus homínidas empezaron a aprender y a modificar su conducta a partir de la experiencia acumulada por los demás miembros del grupo. Fue la emergencia de esta memoria compartida (conductual, lingüística, tecnológica, etc.), así como la identificación de los miembros del grupo a partir de su adecuación a las destrezas transmitidas, lo que marcó la formación de un estilo de vida propiamente humano. Aprender de los demás no es, sin embargo, un fenómeno exclusivo de nuestro linaje y, de hecho, ya se han individuado, especialmente entre los mamíferos y sobre todo los simios, muchas y complejas formas de transmisión ontogénica de conocimientos (de procesos de enseñanza-aprendizaje). Es indicativo, en este sentido, el hecho de que muchos estudiosos del comportamiento animal y de la biología social no dudan en emplear el término «cultura» para describir todas aquellas formas de interacción en las que un organismo aprende a modular su conduta sobre la conducta de sus congéneres.

En nuestra opinión, sin embargo, la transmisión ontogénica de conocimientos es sí imprescindible, pero no suficiente: para que se dé cultura, tal como señala Lotman (1996), también es necesario que el sistema -a partir de sus procesos comunicativos y mnésicos- produzca nueva información, información no disponible ni previsible considerando las estructuras y códigos de partida del sistema. Las conductas que definen a la cultura deben ser, en otros términos, «amorales» (Lotman, 1993). Y esta «amoralidad» no es sino una consecuencia directa de la semiosis, de la emergencia de una nueva complejidad cognoscitiva por la que los procesos significantes se abren, gradual o explosivamente, a nuevas e imprevisibles posibilidades correlativas.

Así pues, si vivir en sociedad significa, fundamentalmente, aprender de los demás (incluso cuando lo que se aprende son los errores, las obsesiones y los miedos de los demás), las dinámicas culturales también incluyen un cuestionamiento intrínseco de lo que se aprende: la transmisión de conocimientos es compleja e imperfecta y produce fluctuaciones que reorganizan constantemente el campo relacional.

16. Me gustaría, antes de concluir, volver a considerar brevemente algunas de las principales implicaciones teóricas del pensamiento sistémico. Sobre todo para conjurar el riesgo de reavivar una vieja pero siempre resur- 
giente disputa: la que opone los reduccionistas a los holistas, los atomistas a los organicistas, los que defienden a ultranza la búsqueda de los causantes últimos e invariables a los que confían ciegamente en la existencia (casi mística) de totalidades indivisibles. Porque el pensaminto sistémico representa, más bien, un intento de superar esta diatriba y sus infinitas (¿y estériles?) polémicas: de hecho, la propia individuación de totalidades y partes y de sus relaciones mutuas ya constituye, de por sí, una cuestión sistémica fundamental.

Claramente, el pensamiento sistémico es inconciliable con cualquier práctica o perspectiva reduccionista, pero que nadie confunda «reducción» con «análisis» (y aún menos con «ciencia»). Rechazar con contundencia la idea de que el operar de un sistema pueda ser explicado completamente a partir de la estructura o función de algunos de sus componentes no significa excluir de nuestra actividad cognoscitiva la búsqueda de elementos y funciones. Tampoco significa negar la existencia de estructuras y procesos invariantes o renunciar a toda pretensión de universalidad. Recordemos que explicar un fenómeno (evento, proceso o estructura) significa, sustancialmente, producir otra descripción de ese fenómeno, una descripción que nos permita integrarlo en un sistema de conocimientos operacional y socialmente válido. Resulta interesante, por lo tanto, y acaso necesario, averiguar qué tipo de descripción se produce en el seno del análisis.

Aun a riesgo de trivializar la cuestión, se puede decir que mientras las explicaciones de tipo reduccionista apuntan a una «simplificación» del fenómeno descrito, en un intento de individuar las especificaciones de un alfabeto minímo (y por lo tanto manipulable) de constituyentes y relaciones fundamentales, las explicaciones sistémicas consisten en una «complejización» del fenómeno, es decir, en un intento de individuar aquellas densidades relacionales que definen al fenómeno en nuestro dominio cognoscitivo. El análisis sistémico, en otros términos, parte del presupuesto (o pre-juicio) de que la individuación de cualquier elemento pertinente en el análisis depende de cómo este elemento se integra en el sistema observado y, por lo tanto, en la red de relaciones en que el sistema participa y deriva, incluida la propia práctica de observación.

Por los mismos motivos, tampoco se puede asimilar el pensamieto sistémico a la tradición filosófica del holismo organicista o vitalista. La noción misma de «totalidad» es, en el discurso sistémico, sumamente relativa, pues depende de las fronteras, los umbrales que el propio observador y su comunidad trazan a lo largo y lo ancho de su red relacional. Una célula, un sistema planetario, la biosfera terrestre, un ecosistema, un ser vivo, un sistema lite- 
rario, una sociedad, una familia, un sistema de producción y consumo, una cultura: todos conjuntos, totalidades, textos que presentan a la mirada del observador interesado determinadas partes y relaciones definitorias; tales partes y relaciones, sin embargo, no existen ni se conocen de por sí, sino que se hallan aglutinadas (organizadas, integradas) en una unidad estructural, funcional y operacional que queda definida por sus dinámicas de estado y de deriva en el dominio de observación.

En último término, las preguntas que justifican y motivan todo procedimiento de análisis son las siguientes: «¿Qué significa este texto?», «¿Cómo funciona?», «¿Cómo está hecho?», «¿Cuál es su posición en el orden que nos rodea y que nos conforma?». Se trata, sin duda alguna, de preguntas legítimas. De pasiones legítimas. Pero mancas (e incluso peligrosas) si no consideramos también esta otra pregunta: «¿Por qué este texto significa, funciona y se presenta y posiciona así para nosotros?». Desde un punto de vista meta-teórico o epistemológico, es esta última pregunta, igual de legítima y «pasional» que las anteriores, la que sostiene el discurso sistémico. ¿Qué es lo que nos motiva y empuja en el análisis? ¿Qué tradición nos valida? ¿Qué tradición validamos? ¿Dé dónde vienen nuestros instrumentos? ¿Cuáles son nuestros objetivos? ¿Qué es lo que olvidamos y qué es lo que creamos en el proceso analítico? Cuestiones (y pasiones) que inevitablemente nos reconducen, lo queramos o no, lo aceptemos o no, seamos o no conscientes de ello, a los derroteros y flujos de la historia y de la interpretación. A los territorios de la cultura. A los ámbitos de interrogación de la semiótica.

\section{REFERENCIAS BIBLIOGRÁFICAS}

BATESON, G. (2000). Verso un'ecologia della mente. Milano: Adelphi.

BOJNIČANOVÁ, R. (2009). «La pervivencia del mito del bandido en el teatro postromántico español, eslovaco y checo». En Jornadas de estudios románicos. Sección de hispanística. Tomo I: literatura, B. Ulašin, S. Vertanová (eds.), 35-50. Bratislava: AnaPress.

CROS, E. (1986). Literatura, ideología y sociedad. Madrid: Gredos.

- (2009). La sociocrítica. Madrid: Arco / Libros.

- (2010). «Sociocrítica e interdisciplinariedad». En Sociocrítica e interdisciplinariedad, A. Chicharro y F. Linares Alés (eds.), 13-24. Granada: Instituto Internacional de Sociocrítica / Ediciones Dauro. 
ECO, U. (1979). Lector en fabula. Milano: Bompiani.

- (1990). I limiti dell'interpretazione. Milano: Bompiani.

- (1997). Kant e l'ornotorinco. Milano: Bompiani.

EVEN-ZOHAR, I. (1990). «Polysystem Theory». Poetics Today. International Journal for Theory and Analysis of Literature and Communication 11.1, 9-26.

- (1999). «Factores y dependencias en la cultura. Una revisión de la Teoría de los polisistemas». En Teoría de los polisistemas: estudio introductorio, M. Iglesias Santos (ed.), 23-52. Madrid: Arco / Libros.

GADAMER, H. G. (1983). Verità e metodo. Milano: Bompiani.

HJELMSLEV, L. (2009). Teoria del linguaggio. Résumé. Vicenza: Terra Ferma.

KUČERKOVÁ, M. (2011). «Isabel Allende e la scrittura come fenomeno culturale». XLinguae.eu. A Trimestral European Scientific Language Review 4, 3-24.

LAMPIS, M. (2011). «La semiotica della cultura come semiotica sistemica». E/C. Rivista on-line dell'AISS Associazione Italiana Studi Semiotici (también en http://www.ec-aiss.it/archivio/tipologico/ricerche.php).

LEVI MONTALCINI, R. (1999). La galassia mente. Milano: Baldini \& Castoldi.

LOTMAN, I. M. (1982). Estructura del texto artístico. Madrid: Istmo.

- (1993). La cultura e l'esplosione. Milano: Feltrinelli.

- (1996). La semiosfera I. Semiótica de la cultura y del texto. Madrid: Cátedra.

- (1998). La semiosfera II. Semiótica de la cultura, del texto, de la conducta y del espacio. Madrid: Cátedra.

MATURANA, R. H. (2009). La realidad: ¿objetiva o construida? Vol. I. Fundamentos biológicos de la realidad. Barcelona: Anthropos.

MORIN, E. (2004). «La epistemología de la complejidad». Gazeta de Antropología 20 (también en http://hdl.handle.net/10481/7253).

PAPPOVÁ, P. (2008). «El dualismo del castellano en La reina del sur de Arturo Pérez-Reverte». En Culture, text, identity, S. Hevešiová, L. Horňáková (eds.), 115-126. Nitra: Filozofická Fakulta UKF. 
ŠTRBÁKOVÁ, R. (2011). «Vestirse como el otro. Ampliación semántica y cambios en el uso del objeto». En Philologia XXI. Supplementum II, 195-207. Bratislava: Univerzita Komenského.

Recibido el 6 de junio de 2012.

Aceptado el 30 de septiembre de 2012. 